Article original

\title{
Radiothérapie conformationnelle du cancer de la prostate non métas- tatique : Experience du service de radiothérapie de l'EHS en Oncologie Emir Abdelkader d'Oran
}

\author{
Conformational radiotherapy of no metastatic prostate cancer: Experience of the radia- \\ tion therapy department of EHS Oncology Emir Abdelkader of Oran
}

\author{
Lotfi Taleb ${ }^{1,4}$, Karima Chenni ${ }^{3}$, Ismail Zergoug ${ }^{1}$, Abdelbaki Boukerche ${ }^{1,3}$, Ahmed Fethi Dali-Youcef 2,4 \\ 1 Service de Radiothérapie - EHS en Oncologie Emir Abdelkader d'Oran \\ ${ }^{2}$ Service de Radiothérapie - CHU Oran \\ ${ }^{2}$ Service de Biostatistique et d'Epidémiologie Clinique d'Oran \\ ${ }^{4}$ Faculté de Médecine, Université d'Oran 1
}

\section{MOTS CLÉS}

Cancer de la prostate; PSA; Diagnostic; Radiothérapie conformationnlle.

\begin{abstract}
Résumé
Introduction - Le cancer de la prostate est devenu au cours de ces dernières décennies un véritable problème de santé publique, par sa fréquence et sa gravité. La radiothérapie conformationnelle constitue aujourd'hui la technique standard dans l'irradiation des cancers non métastatiques de la prostate en raison du bénéfice apporté en termes de tolérance et de contrôle de la maladie par rapport à une radiothérapie conventionnelle $2 \mathrm{D}$. L'objectif de notre travail est d'étudier la survie sans rechute biochimique et la survie globale des patients atteints d'un cancer non métastatique de la prostate, traités au service de radiothérapie de l'EHS en oncologie « Emir Abdelkader » d'Oran, durant la période de juin 2010 à décembre 2014.
\end{abstract}

Patients et méthodes - Il s'agit d'une étude prospective de type descriptif. Au cours de la période de l'étude, quatre-vingt-dix patients $(n=90)$ atteints d'un cancer de la prostate non métastatique traités par radiothérapie conformationnelle ont été colligés.

Résultats - L'âge moyen de de nos patients était de 66,3 ans. Le cancer de la prostate était diagnostiqué à un stade localement avancé et/ou localisé à haut risque selon la classification de D'AMICO chez $80 \%$ des cas. La radiothérapie était exclusive chez 80 patients, postopératoire immédiate chez cinq patients et de rattrapage chez cinq cas. Une hormonothérapie était associée à la radiothérapie chez la majorité des patients soit $94,4 \%$ des cas. Avec un suivi médian de 31 mois; la probabilité de survie sans rechutes biochimiques est de $64 \%$ alors que la survie globale est

'Auteur correspondant: lotaleb@yahoo.fr 


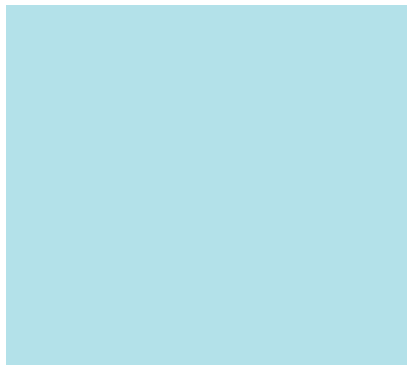

\section{KEY WORDS}

Prostate cancer; PSA; Diagnostic; Conformal radiotherapy.

\section{Introduction}

Le cancer de la prostate est le cancer le plus fréquent chez l'homme de plus de 50 ans dans le monde, avec plus de 670 000 nouveaux cas diagnostiqués annuellement et la 3ème cause de mortalité par cancer après le cancer du poumon et le cancer colorectal [1].

En Algérie, il occupe le quatrième rang selon les différents registres du cancer [2]. Il est devenu un problème majeur de santé publique. Son incidence est en augmentation, elle concerne les cancers de la prostate localisés, le plus souvent asymptomatiques et s'explique principalement par deux facteurs, qui sont l'allongement de la durée de vie et le dépistage individuel par le dosage de l'Antigène Spécifique de Prostate (PSA) [3]. Les biopsies prostatiques souvent échoguidées sont l'examen standard qui permet d'établir le diagnostic de cancer de la prostate et d'apporter les critères histo-pronostiques [4].

Le contrôle local est l'un des éléments les plus importants dans l'évaluation des résultats du traitement des cancers de prostate [5]. La Radiothérapie occupe une place centrale dans le traitement du cancer de la prostate tous stades confondus à la fois en cas de tumeur en place et après prostatectomie radicale en situation adjuvante ou de récidive biochimique [6]. Les progrès de l'informatique et de l'imagerie des an- 
nées 1990 ont fait évoluer la radiothérapie conventionnelle $2 \mathrm{D}$ qui basait ses faisceaux sur des repères osseux en radiothérapie conformationnelle 3D, substituant aux deux dimensions du plan le volume [7]. La radiothérapie conformationnelle représente une avancée majeure dans le traitement du cancer de la prostate, tant sur le plan de la tolérance grâce à une meilleur protection des organes à risque qu'en termes d'efficacité. Elle est devenue une alternative à la chirurgie et doit être proposée à tout patient porteur d'un cancer de prostate localisé de risque faible ou intermédiaire [8]. C'est le traitement de référence des formes localement évoluées associée à une hormonothérapie longue [9].

Notre étude a pour objectif d'étudier la survie sans rechute biochimique et la survie globale des patients atteints d'un cancer non métastatique de la prostate, traités par radiothérapie conformationnelle durant la période de juin 2010 à décembre 2014 à l'Etablissement Hospitalier Spécialisé (EHS) en Oncologie Emir Abdelkader d'Oran.

\section{Patients et méthodes}

\section{Type d'étude}

Il s'agit d'une étude prospective de type descriptif réalisée au service de radiothérapie de l'EHS en oncologie Emir Abdelkader de Juin 2010 à Décembre 2014 et portant sur des patients âgés de moins de 80 ans atteints d'un adénocarcinome de la prostate prouvé histologiquement, localisé ou localement avancé, sans adénopathies pelviennes et sans métastases osseuses ou viscérales. A l'admission, tous les patients ont bénéficié d'un examen clinique comportant un toucher rectal, un examen biologique incluant le taux sérique de prostate specific antigen (PSA), un examen anatomopathologique de la tumeur utilisant le score de Gleason, et un bilan d'extension basé essentiellement sur une IRM pelvienne et une scintigraphie osseuse. Au terme de ce bilan, l'extension tumorale a été classée selon la classification TNM de 2010 [10], et la prise en charge thérapeutique a été orientée selon la classification pronostique de D'Amico [11].

\section{Schéma thérapeutique}

La technique d'irradiation conformationnelle tridimensionnelle a été utilisée dans trois situations distinctes: exclusive chez les patients non opérés, en post-opératoire adjuvante immédiate chez les patients présentant des facteurs pronostiques péjoratifs en post-opératoires (extension extra-capsulaire de la maladie T3a, un envahissement des vésicules séminales T3b, des tranches de section chirurgicales atteintes R1, un score de Gleason $>7$ ) et après une prostatectomie radicale en situation de rattrapage lors d'une récidive biochimique (réascension de la concentration sérique de PSA supérieur à
$0,2 \mathrm{ng} / \mathrm{ml}$ après une période d'indétectabilité).

La préparation de l'irradiation a utilisé un scanographe simulateur dédié à la radiothérapie afin de déterminer les volumes cibles à irradier selon les recommandations de l'European Organization on treatment of cancer (EORTC) [12]. Ces volumes cibles sont la prostate ou loge prostatique, les vésicules séminales, les aires ganglionnaires pelviennes et les organes à risque (vessie, rectum, intestin grêle, têtes fémorales et bulbe pénien).

L'inclusion des aires ganglionnaires pelviennes dans le volume cible était indiquée pour les patients ayant un risque d'atteinte de ces ganglions supérieur à $15 \%$ selon la formule de Roach [13]. Les doses délivrées sont de 46 Gy au niveau du pelvis, de 70 à 74 Gy au niveau de la prostate et de 64 à 66 au niveau de la loge prostatique, en fractionnement et étalement classique à raison de 2 Gy par séance, cinq séances par semaine, avec un respect strict des contraintes de doses aux organes à risque. Les contraintes de dose sont issues du guide de procédure de radiothérapie externe 2007 [14]. L'irradiation était délivrée par des accélérateurs linéaires de haute énergie de 18MV, équipés de collimateur multilames. Une privation androgénique de type analogue de la LH-RH associée à la radiothérapie, est administrée pour une durée de six à neuf mois chez les patients ayant un cancer de risque intermédiaire selon la classification pronostique de D'Amico, alors qu'elle est de trois ans pour les formes à haut risque.

Le suivi des patients après la fin du traitement était uniquement clinique et biologique. Le dosage du PSA total permet de suivre le statut carcinologique du patient. Aucun examen d'imagerie n'est recommandé en l'absence de récidive biologique. Le rythme du suivi est de trois mois après la fin du traitement puis tous les six mois jusqu'à cinq ans puis annuel.

L'efficacité du traitement a été évaluée par deux paramètres qui sont:

- La survie sans rechute biochimique définie selon les critères de Phoenix: une augmentation du taux de PSA sérique de 2 $\mathrm{ng} / \mathrm{ml}$ au-dessus du nadir absolu (sans valeur seuil du nadir) après traitement [15]. Cette augmentation sera contrôlée par un nouveau dosage du PSA réalisé à trois mois d'intervalle dans le même laboratoire. La date du premier dosage sera retenue comme date de progression si cette augmentation est confirmée.

- La survie globale (décès quelle que soit la cause).

\section{Méthode statistique}

Les données quantitatives sont exprimées par la moyenne, l'écart-type, l'étendue et la médiane. Les données qualitatives sont exprimées en pourcentage. La durée de survie sans 
récidive biochimique est définie par le temps écoulé entre la date du début de la radiothérapie et la date de survenue d'une récidive biochimique. Les courbes de survies (survie sans rechute biochimique et survie globale) ont été obtenues par la méthode de Kaplan-Meier. L'analyse statistique des données a été faite par le logiciel Epi-info version 3.5.3 January 26, 2011 et par le logiciel SPSS version 21.0.

\section{Considérations éthiques}

Le patient est informé du choix de l'indication, du plan de traitement, son déroulement, sa durée ainsi que les effets secondaires prévisibles. Le consentement verbal du patient a été obtenu avant le début du traitement. Les données des patients ont été anonymisées.

\section{Résultats}

$\mathrm{Au}$ total quatre-vingt-dix patients ont été inclus dans

Tableau 1 : Caractéristiques diagnostiques et thérapeutiques des patients $(n=90)$

\begin{tabular}{|c|c|c|}
\hline & $\mathrm{n}$ & $\%$ \\
\hline \multicolumn{3}{|l|}{ Dépistage } \\
\hline Oui & 15 & 17 \\
\hline Non & 75 & 83 \\
\hline \multicolumn{3}{|l|}{ Nature du prélèvement } \\
\hline Biopsies prostatiques & 87 & 96,7 \\
\hline Résection transurétrale de la prostate & 3 & 3,3 \\
\hline \multicolumn{3}{|l|}{ Tumeur T UICC (2010) } \\
\hline T1 & 2 & 2,2 \\
\hline T2 & 39 & 43,3 \\
\hline T3-T4 & 49 & 54,5 \\
\hline \multicolumn{3}{|l|}{ PSA (ng/ml) } \\
\hline$\leq 10$ & 16 & 17,8 \\
\hline $10-20$ & 37 & 41,1 \\
\hline$>20$ & 37 & 41,1 \\
\hline \multicolumn{3}{|l|}{ Score de Gleason } \\
\hline 6 & 48 & 53,3 \\
\hline 7 & 23 & 36,7 \\
\hline$>7$ & 9 & 10 \\
\hline \multicolumn{3}{|l|}{ Groupe pronostique de D'Amico } \\
\hline Favorable & 2 & 2,2 \\
\hline Intermédiaire & 16 & 17,8 \\
\hline Défavorable & 72 & 80 \\
\hline \multicolumn{3}{|l|}{ Prostatectomie } \\
\hline Oui & 10 & 11 \\
\hline Non & 80 & 89 \\
\hline \multicolumn{3}{|l|}{ Type de radiothérapie } \\
\hline Exclusive & 80 & 89 \\
\hline Adjuvante & 5 & 5,5 \\
\hline De rattrapage & 5 & 5,5 \\
\hline \multicolumn{3}{|l|}{ Irradiation pelvienne } \\
\hline Oui & 63 & 70 \\
\hline Non & 27 & 30 \\
\hline \multicolumn{3}{|l|}{ Hormonothérapie } \\
\hline Oui & 85 & 94,5 \\
\hline Non & 5 & 5,5 \\
\hline
\end{tabular}

l'étude, originaires essentiellement de la wilaya d'Oran $(51,1 \%)$ suivie de la wilaya de Tlemcen $(15,6 \%)$. L'âge médian des patients était de 66,5 ans avec des extrêmes allant de 52 à 78 ans. Les circonstances de découverte étaient dominées par les signes du bas appareil urinaire chez $83 \%$ des patients ; cependant chez $17 \%$ des cas, le cancer de la prostate a été découvert fortuitement par le dosage du PSA, dans le cadre du dépistage individuel. Le taux du PSA total avant le diagnostic était élevé $(>4 \mathrm{ng} / \mathrm{ml}$ ) chez tous les patients, avec une moyenne de $24,4 \pm 19,6 \mathrm{ng} / \mathrm{ml}$ et des extrêmes allant de 6 à $108 \mathrm{ng} / \mathrm{ml}$. Le taux médian du PSA total était de $17,5 \mathrm{ng} / \mathrm{ml}$.

Dans notre série, le diagnostic de cancer de la prostate était affirmé sur les résultats anatomopathologiques de la ponction biopsie de prostate transrectale écho-guidée chez $96,7 \%$ des patients et lors d'une résection trans-urétrale de prostate RTUP chez seulement trois patients (3.3\%). Le score de Gleason était de 6, 7 et supérieur à 7 chez respectivement $53,3 \%, 36,7 \%$ et $10 \%$ des patients. Les tumeurs appartiennent, pour leur grande majorité $(80 \%)$ au groupe pronostique défavorable ( $\geq T 2 \mathrm{c}, \mathrm{G} \geq 8, \mathrm{PSA}>20 \mathrm{ng} / \mathrm{ml}$ ), $17,8 \%$ au groupe pronostique intermédiaire (T2b, $\mathrm{G}=7, \mathrm{PSA}=10-20 \mathrm{ng} /$ $\mathrm{ml}$ ) tandis que le groupe favorable (T2a, G = 6, PSA < 10ng/ $\mathrm{ml}$ ) n'a représenté que 2,2\% soit deux cas.

La majorité des patients soit $94,4 \%$ ont bénéficié d'une hormonothérapie néoadjuvante et concomitante à la radiothérapie de type analogue LH-RH. La durée globale de l'hormonothérapie était variable en fonction du stade de la maladie. Elle était administrée pour une courte durée ( $\leq 9$ mois) dans les formes à risque intermédiaire chez 17 patients (20\%) et pour une longue durée ( $\geq 30$ mois) dans les formes à haut risque chez 68 patients $(80 \%)$.

Tous les patients ont bénéficié d'une radiothérapie conformationnelle qui était exclusive chez 80 cas, post-opératoire adjuvante immédiate chez cinq patients et en situation de rattrapage après une récidive biochimique chez cinq patients également. En radiothérapie exclusive, le volume cible irradié comprenait la prostate, les vésicules séminales et les ganglions pelviens chez 56 patients (70\%), alors qu'il était limité à la prostate et les vésicules séminales chez 24 patients (30\%). En radiothérapie post-opératoire, le volume cible incluait la loge prostato-séminale (incluant le col de la vessie, l'anastomose urétro-vésicale et l'urètre) et les ganglions pelviens chez sept patients, alors qu'il était limité à la seule loge prostatique chez trois patients. Chez ces derniers, le curage ganglionnaire pelvien était suffisant $(\geq 10$ ganglions) et revenant négatif ( $\mathrm{pN} 0$ ). Les caractéristiques des patients sont présentées dans le tableau 1.

La durée moyenne de la prise en charge radiothérapique dans notre population d'étude a été estimée à $51,6 \pm 10,2$ 
jours. Le suivi médian de notre population d'étude est de 31 mois avec des extrêmes allant de 13 à 66 mois. Aucun patient n'était perdu de vue au cours de cette période. La réponse biologique au traitement était bonne puisque le PSA de 89 patients soit $98.9 \%$ a été normalisé (PSA nadir $<1 \mathrm{ng} / \mathrm{ml}$ ). La durée moyenne d'atteinte du PSA nadir était de 8,6 $\pm 4,6$ mois avec des extrêmes allant de 3 à 22 mois. Le délai médian de survenue de la rechute biochimique était de 39,4 mois avec des extrêmes allant de 15 à 55 mois.

La probabilité de survie sans rechute biochimique à 3 ans selon les critères de Phoenix 2006 est de 94,5\% [IC à 95\%: 91,2 - 97,43], alors qu'à 5 ans elle est de $64 \%$ [IC à 95\%: $53,65-74,26]$. La probabilité de survie globale de notre population d'étude à cinq ans était estimée à 93,7\% [IC à 95\%: $86,05-97,51]$ voir figure 1 et figure 2 .

\section{Discussion}

Le cancer de la prostate est un cancer du sujet âgé. Dans

Figure 1: Courbe de survie sans récidive biochimique de la population d'étude durant la période de juin 2010 à décembre2014. Service de radiothérapie, EHSO Emir Abdelkader d'Oran

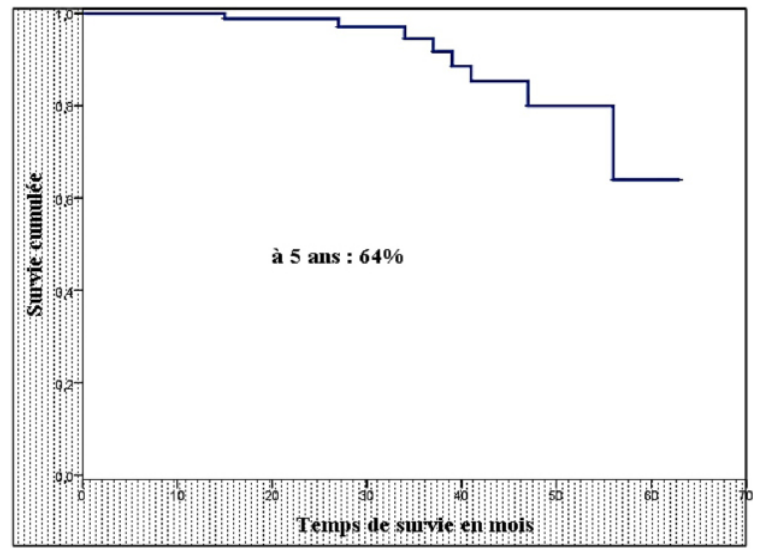

Figure 2 : Courbe de survie globale de notre population durant la période de juin 2010 à décembre 2014. Service de radiothérapie, EHSO Emir Abdelkader d'Oran.

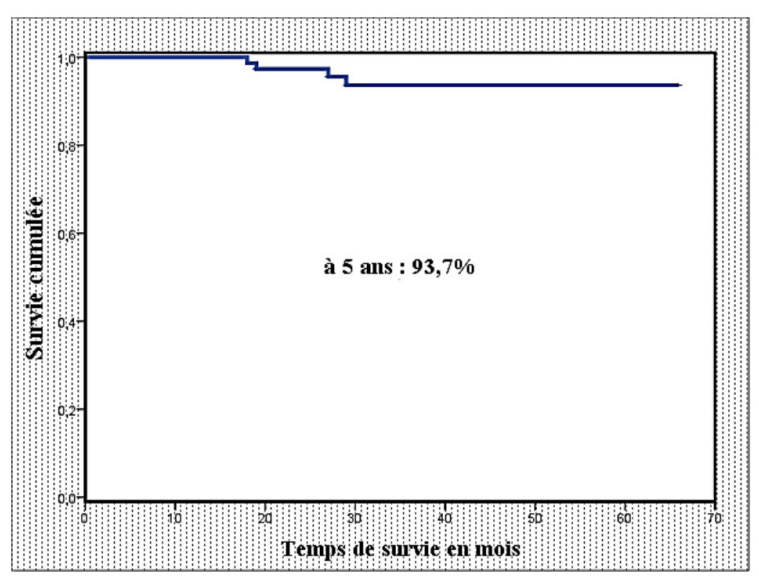

notre série, l'âge médian est de 66,5 ans ; alors que dans l'étude d'Ayad (Blida) et celle d'El azhari (Rabat), il était respectivement de 69 ans et 67 ans $[16,17]$. Il est relativement inférieur à celui retrouvé chez les occidentaux, notamment en France (66,5 ans vs 71 ans) [18]. Cette disparité peut être expliquée par le fait que dans notre pratique, les sujets âgés sont mis d'emblée sous hormonothérapie, souvent en raison de leurs comorbidités associées.

Les signes du bas appareil urinaire (SBAU) ont constitué le motif principal de consultation et de découverte du cancer de la prostate chez nos patients $(83,3 \%$ ) témoignant du stade avancé de la maladie. Ces résultats rejoignent ceux des séries maghrébines et africaines où le cancer est découvert à un stade tardif $[19,20]$. Cependant en occident, le cancer de la prostate est diagnostiqué chez des patients asymptomatiques lors du dépistage individuel dans $80 \%$ des cas [21].

En termes de prise en charge, l'hormonothérapie basée sur un analogue de la LH-RH, a été associée à la radiothérapie dans $94,4 \%$ des cas. Cette large prescription était en conformité avec les consensus de la littérature en présence des critères d'agressivité (Gleason supérieur ou égal à 7, effraction capsulaire, atteinte des vésicules séminales, etc.) et ceci afin d'améliorer l'efficacité de la radiothérapie.

Plusieurs essais ont démontré le bénéfice de l'association de l'hormonothérapie à la radiothérapie en termes de survie sans récidive biochimique, de récidive locale ou à distance et de mortalité spécifique; et ceci aussi bien dans les formes à risque intermédiaire pour une courte durée de 6 mois [22,23] que dans les formes à haut risque pour une longue durée $\geq 30$ mois $[24,25,26,27]$.

Concernant la prise en charge radiothérapique, nos choix techniques se sont basés sur une large revue des différentes données scientifiques concernant chaque étape dans le processus de la radiothérapie conformationnelle; et sur ce que nous offrait notre plateau technique comme moyens [28, 29].

La détermination des volumes cibles étaient adaptée au risque pronostique du cancer de la prostate selon la classification de D'Amico [11]. L'irradiation a concerné les aires ganglionnaires pelviennes dans $62,2 \%$ des cas vs $16,8 \%$ dans l'étude de Ponvert [30]. Ceci est en rapport avec la fréquence élevée des formes à haut risque.

Pour les doses d'irradiation, plusieurs études randomisées ont montré le bénéfice de l'escalade de dose (78 à 80Gy) dans le cancer de la prostate, en termes de survie sans récidive biologique et clinique mais sans amélioration de la survie globale, par rapport aux doses conventionnelles (68 à 70Gy) [31, 32, 33, 34,35]. Dans ces études, la technique d'irradiation utilisée était conformationnelle par faisceaux 
statiques sans modulation d'intensité avec un taux élevé de la toxicité rectale le plus souvent $[31,32,33]$ et vésicale $[35,36]$.

Par principe de précaution, dans notre pratique nous avons choisi des doses d'irradiation intermédiaires; dans le cas de la radiothérapie exclusive ; avec un respect strict des contraintes de doses aux organes à risque [14].

Les doses délivrées étaient respectivement 70 Gy ,72 Gy et 74 Gy chez $44,4 \%, 25,6 \%$ et $18,9 \%$ vs $73,3 \%, 5,3 \%$ et $5,3 \%$ dans l'étude d'Elie Nasr [37] alors qu'elle était de 70 Gy chez $78,2 \%$ des patients dans l'étude d'El Marjany [38].

Dans notre étude, la probabilité de survie sans récidive biochimique à 5 ans est de $64 \%$. Elle est comparable à celle retrouvée par Bolla (75\%), Mottet (64.,7\%) et Afshin (65.,1\%) [39, 40,41]. En revanche elle est inférieure à celle d'El Marjany estimée à $94 \%$ [38]; série dans laquelle, le nombre de patients ayant bénéficié d'une radiothérapie adjuvante ou de rattrapage est plus important que dans notre série (22\% vs $11 \%$ ). En termes de survie globale, elle est de $93,7 \%$, rejoignant les résultats de Ponvert et d'El Marjany estimés à $94 \%[30,38]$. D'autre part, les études de Bolla et Hanks ont révélé des probabilités de survie respectives de $78 \%$ et $80 \%$ $[39,42]$. Cependant, il est important de noter certaines limites à notre étude.

Il s'agit en premier lieu de la prise en charge des patients qui n'a pas été décidée en réunion de concertation pluridisciplinaire (RCP) d'onco-urologie. Cette dernière n'a été lancée qu'en 2015 dans un des deux établissements hospitalo-universitaires d'Oran. D'autre part, la taille de l'échantillon était limitée du fait que les urologues libéraux susceptibles d'orienter les patients n'ont été informés qu'en 2012, du lancement de cette technique de radiothérapie conformationnelle à l'EHS en oncologie Emir Abdelkader d'Oran.

\section{Conclusion}

Le cancer de la prostate est la troisième cause de mortalité par cancer chez l'homme. Sa prise en charge devient un enjeu de santé publique majeur en raison du vieillissement de la population.

Actuellement la radiothérapie conformationnelle s'inscrit comme une des grandes options curatrices du traitement de cette affection au stade localisé ou locorégional. Elle permet d'offrir des résultats carcinologiques comparables à celles de la prostatectomie radicale. Malgré un recul médian moins important que dans les séries occidentales, les résultats thérapeutiques de notre étude en termes de survie sans rechute biochimique et de survie globale sont satisfai- sants. Une actualisation ultérieure des résultats sera toutefois indispensable, compte tenu d'un recul encore limité et de l'évolution naturellement lente du cancer de la prostate. Par ailleurs, la promotion des réunions de concertation pluridisciplinaires est nécessaire pour optimiser la prise en charge des patients.

\section{Conflits d'intérêt}

Les auteurs déclarent n'avoir aucun conflit d'intérêt.

\section{Références bibliographiques}

[1] Wein A, Kavoussi L, Partin A, Peters C. Campbell-Walsh Urology. 10th edition review. International edition. January 2012 Pathophysiology of Urinary Tract Obstruction (1087-1121), ISBN: 978-0-80892439-5.

[2] Ministère de la Santé de la Population et de la Réforme Hospitalière ; Direction Générale des Structures de Santé: Manuel de Prise en Charge Du Cancer de la Prostate Février 2016.www.sante.dz/ cancer/Prostate

[3] Haute Autorité de Santé, Guide ALD n ${ }^{\circ}$ 30: Cancer de la prostatejanvier 2012.

[4] Epstein JI, Srigley J, Grignon D, Humphrey P. Recommandations for the reporting of prostate carcinoma: Association of Directors of Anatomic and Surgical Pathology. Am J Clin Pathol 2008; 129:24-30.

[5] Chauvet. B, Alfonsi. M, Rauglaudre. G, Reboul F. Cancers de prostate : le traitement local par irradiation a-t-il un impact sur la survie ? Cancer/Radiother $2002 ; 6$ : 141-6.

[6] Maingon P, Bolla b M., Truc G, Bosset M, Peignaux K, Ammor A. La radiothérapie de conformation avec et sans modulation d'intensité dans le traitement du cancer localisé de la prostate. Cancer/Radiothérapie 9 (2005) 382-387.

[7] Gross E. Radiothérapie conformationelle du cancer de prostate. Progrès en urologie (2011) 21, 801-807.

[8] Hennequin C, Quero L, Soudi H, Sergent G, Maylin C. Radiothérapie conformationnelle du cancer de la prostate: technique et résultats. EMC Urologie 2006 ; 18-561.

[9] Khalifa. J, Commandeur. F,. Bachaud. J.-M, De Crevoisier. R. Radiothérapie conformationnelle prostatique : quelles marges ? Cancer/Radiothérapie 17 (2013) 461-469.

[10] American Joint Committee on Cancer: Cancer staging Handbook. Seventh ed Springer Eds 2010; 525:34.

[11] D'Amico AV, Whittington R, Malkowicz SB, Schultz D, Blank K, Broderick GA, et al. Biochemical outcome after radical prostatectomy, external beam radiation therapy, or interstitial radiation therapy for clinically localized prostate cancer. JAMA 1998; 280: 969-74.

[12] Boehmer D, Maingon P, Poortmans P, Baron MH, Miralbell R, Remouchamps V, et al. Guidelines for primary radiotherapy of patients with prostate cancer. Radiother Oncol 2006; 79:259-69.

[13] Roach M, Marquez C, Yuo HS, Narayan P, Coleman L, Nseyo

6 JFMO : Nº , Juin 2017 
UO, et al. Predicting the risk of lymph node involvement using the pre-treatment prostate specific antigen and Gleason score in men with clinically localized prostate cancer. Int J Radiat Oncol Biol Phys 1994; 28:33-7.

[14] Société française de radiothérapie externe. Guide des procédures de radiothérapie externe 2007. Cancer Radiother 2008; 12:141314.

[15] Salomona L, Bastide C, Beuzeboc P, Cormier L, Fromont G, Hennequin C, Mongiat-Artus P, Peyromaure M, Ploussard G, Renard-Penna R, Rozet F, Azria.D, Coloby P, Molinié V, Ravery. V, Rebillardb X, Richaud $P$, Villers A, Soulié $M$ et les membres du CCAFU. Recommandations en onco-urologie 2013 du CCAFU: Cancer de la prostate. Progrès en Urologie (2013), Suppl. 2 S69-S102

[16] M. Ayad, L. Abbas, S. Mesli, K. Boualga. La radiothérapie conformationnelle dans le cancer de la prostate : expérience du service de radiothérapie oncologique de Blida, Algérie. Cancer/Radiothérapie 14 (2010) 584-665.

[17] El azhari Jawad. Le cancer de la prostate à l'hôpital militaire d'instruction Mohamed $V$ : profil épidémiologique, aspects clinico-histologiques et thérapeutiques (à-propos de 568 cas). Thèse de Doctorat en Médecine ANNEE: 2009.

[18] Haute Autorité de Santé. Détection précoce du cancer de la prostate; Actualisation du référentiel de pratiques de l'examen périodique de santé (EPS) Mai 2013.

[19] HAJAR Habibi. La Corrélation Entre Le score de Gleason de la biopsie et celui de la pièce de prostatectomie radicale (A propos de 47 cas).THESE de Doctorat en Médecine. Année 2015.

[20] Gueye SM., Jalloh M., Labou I., Niang L., Kane R., Ndoye M. Profil clinique du cancer de la prostate au Sénégal. African Journal of Urology, Vol. 10, No. 3 (2004).

[21] Andriole GL, Crawford ED, Grubb RL 3rd, Buys SS, Chia D, Church TR, et al. PLCO Project Team. Prostate cancer screening in the randomized Prostate, Lung, Colorectal, and Ovarian Cancer Screening Trial: mortality results after 13 years of follow- up. J Natl Cancer Inst 2012; 104:125-32.

[22] D’Amico A, Manola J, Loffredo M, Renshaw A, Dellacroce A, Kantoff P. 6-month suppression plus radiation therapy vs radiation therapy alone for patients with clinically localized prostate cancer. JAMA 2004; 292:821-7.

[23] Jones CU, Hunt D, Mc Gowan DG, Amin MB, Chetner MP, Bruner DW, et al. Radiotherapy and short- term androgen deprivation for localized prostate cancer. N Engl J Med 2011; 365:107-18.

[24] Souhami L, Bae K, Pilepich M. Impact of the duration of adjuvant hormonal therapy in patients with locally advanced prostate cancer treated with radiotherapy: a secondary analysis of RTOG 85-31. J Clin Oncol 2009; 27:2137-43.10.

[25] D'Amico A.V: Combined modality staging for clinically localized adenocarcinoma of the prostate as the basis for patient selection for randomised trials of neoadjuvant chemotherapy. Urologic Oncology, 2001; 6: 171-172.

[26] Lawton CA1, De Silvio M, Roach M 3rd, Uhl V, Kirsch R, Seider M, Rotman M, Jones C, Asbell S, Valicenti R, Hahn S, Thomas CR Jr. An update of the phase III trial comparing whole pelvic to prostate only radiotherapy and neoadjuvant to adjuvant total androgen suppression: updated analysis of RTOG 94-13, with emphasis on unexpected hormone/radiation interactions. Int J Radiat Oncol Biol Phys. 2007 Nov 1; 69 (3):646-55.

[27] Bolla M, Collette L, Blank L, Warde P, Dubois JB, Mirimanoff RO, et al. Long-term results with immediate androgen suppression and external irradiation in patients with locally advanced prostate cancer (an EORTC study): a phase III randomised trial. Lancet 2002; 360(9327):103-6.

[28] Barret A, Dobbs J, Morris S, Roques T. Practical Radiotherapy Planning, 4th edition, chapter 28, 2009 pp 332-50.

[29] Halperin E, Perez C, Brady L. Perez and Brady's Principles and Practice of Radiation. Oncology 5th Edition: 2008; 1484-502.

[30] Pontvert D, Gaboriaud G, Flam T, Jourdan-Da Silva N, Thiounn N, Mammar H, Beuzeboc P, Debré B. Radiothérapie conformationnelle à $76 \mathrm{~Gy}$ des cancers localisés de la prostate. Modalités thérapeutiques et résultats préliminaires. Cancer/Radiothérapie 12 (2008) 78-87.

[31] PollackA, Zagars GK, Smith LG, et al. Preliminary results of a randomized radiotherapy dose-escalation study comparing 70 Gy with 78 Gy for prostate cancer. J Clin Oncol 2000; 18:3904-3911.

[32] Zietman AL, De Silvio ML, Slater JD, et al. Comparison of conventional-dose vs high-dose conformal radiation therapy in clinically localized adenocarcinoma of the prostate: a randomized controlled trial. Jama 2005; 294:1233-1239.

[33] Dearnaley DP, Hall E, Lawrence D, et al. Phase III pilot study of dose escalation using conformal radiotherapy in prostate cancer: PSA control and side effects. Br J Cancer 2005; 92:488-498.

[34] Peeters ST, Heemsbergen WD, Koper PC, et al. Dose-response in radiotherapy for localized prostate cancer: results of the Dutch multicenter randomized phase III trial comparing 68 Gy of radiotherapy with 78 Gy. J Clin Oncol 2006; 24:1990-1996.

[35] Beckendorf V, Guerif S, Le Prise E, et al. The GETUG 70 Gy vs. 80 Gy randomized trial for localized prostate cancer: feasibility and acute toxicity. Int J Radiat Oncol Biol Phys 2004; 60:1056-1065.

[36] Dearnaley DP, Sydes MR, Graham JD, Aird EG, Bottomley D, Cowan $\mathrm{RA}$, et al. Escalated-dose versus standard-dose conformal radiotherapy in prostate cancer: first results from the MRC RT01 randomised controlled trial. Lancet Oncol 2007; 8:475-87.

[37] Elie NASR, Sleiman MERHEJ, Dolly NEHME NASR, Georges FARES, Radiothérapie conformationnelle dans le traitement du cancer de la prostate.Evaluation de la toxicité aiguë chez 131 patients Progrès en Urologie (2005), 15, 36-39.

[38] El Marjany M, Bazine A, Lalya I, Zaghba N, Andaloussi K, Sifat H, Hadadi $K$, Mansouri $\mathrm{H}$. La radiothérapie conformationnelle tridimensionnelle dans le traitement du cancer localisé de la prostate : expérience du service de radiothérapie de l'hôpital militaire d'instruction Mohamed V, Rabat, Maroc. J. Afr. Cancer (2014) 6:209-214.

[39] Bolla M, Collette L, Blank L, Warde P, Dubois JB, Mirimanoff RO, et al. Long-term results with immediate androgen suppression and external irradiation in patients with locally advanced prostate cancer (an EORTC study): a phase III randomised trial. Lancet 2002; 360(9327):103-6.

[40] Mottet N, Peneau M, Mazeron JJ, Molinie V, Richaud P. Addition of radiotherapy to long term androgen deprivation in locally advanced prostate cancer: an open randomised phase 3 trial. Eur Urol 2012;62:213-9

[41] Afshin Rakhsha, Amir Shahram, Yousefi Kashi, Bahram Mofid, and 
Mohammad Houshyari. Biochemical progression-free survival in localized prostate cancer patients treated with definitive external beam radiotherapy. Electron Physician. 2015 Oct; 7(6): 1330-1335

[42] Hanks GE, Pajak TF, Porter A, Grignon D, Brereton H, Venkatesan
V,et al. Phase III trial of long-term adjuvant androgen deprivation after neoadjuvant hormonal cytoreduction and radiotherapy in locally advanced carcinoma of the prostate: the Radiation Therapy Oncology

Group Protocol 92-02. J Clin Oncol 2003; 21(21):3972-8 